\title{
Effects of Body Weight on the Safety of High-dose Donepezil in Alzheimer's Disease: Post-hoc Analysis of a Multicenter, Randomized, Open-label, Parallel Design, Three-arm Clinical Trial
}

\author{
Yun Jeong Hong \\ Uijeongbu Saint Mary's Hospital https://orcid.org/0000-0002-4996-4981 \\ Hyun Jeong Han \\ Myongji Hospital, Hanyang University College of Medicine \\ YoungChul Youn \\ Chungang University Medical Center: Chung Ang University Hospital \\ Kyung Won Park \\ Dong-A University Medical Center \\ Dong Won Yang \\ Catholic University of Korea School of Medicine \\ SangYun Kim \\ Seoul National University Bundang Hospital \\ Hwa Jung Kim \\ Asan Medical Center \\ Hyung-Ji Kim \\ Asan Medical Center \\ Miseon Kwon \\ Asan Medical Center \\ Jae-Hong Lee ( $\square$ jhlee@amc.seoul.kr) \\ University of Ulsan College of Medicine
}

Research article

Keywords: Alzheimer's disease, High dose donepezil, Safety, Body weight, Adverse events, Dose-titration

Posted Date: September 21st, 2020

DOl: https://doi.org/10.21203/rs.3.rs-72249/v1

License: (9) (1) This work is licensed under a Creative Commons Attribution 4.0 International License. Read Full License 


\section{Abstract}

Background: Donepezil 23mg is considered for Alzheimer's disease (AD) to optimize cognitive benefits; however, this increases adverse events (AEs), which can negatively influence drug adherence. We investigated whether baseline body weight (BW) differs based on the presence of AEs, and which factors were relevant to the safety of high-dose donepezil.

Methods: This study was a post-hoc analysis of a multicenter randomized trial between 2014 and 2016 . We included patients with moderate to severe $A D$ treated with donepezil $10 \mathrm{mg} /$ day and the daily dose was escalated to $23 \mathrm{mg}$ with/without dose titration. Dose titration indicates $15 \mathrm{mg} /$ day of donepezil before the escalation or $10 \mathrm{mg}$ and $23 \mathrm{mg} /$ day on alternate days before the escalation during the first 4 weeks. The patients were divided into two groups according to the occurrence of AEs of special interest (AESIs) to compare baseline characteristics. We also assessed relationships between BW and AESIs.

Results: Among 160 safety set population, baseline BWs were different between the AESI (+) (n=67) and AESI (-) (n=93) groups. Baseline BW was inversely correlated with the occurrence of AESIs $(p=0.020)$, and this relationship was more prominent in the no-dose titration group $(p=0.009)$ and disappeared in the dose titration groups $(p>0.05)$.

Conclusions: BW was the most important factor which correlated with cholinergic AEs. Hence, stepwise dose-titration should be considered, particularly in patients with low BW, to minimize the inverse relationship between BW and AEs occurrence. ('Clinicaltrials.gov' number NCT02550665 registered on Sep. 15, 2015)

\section{Background}

In moderate to severe Alzheimer's disease (AD), a higher dose of acetylcholinesterase inhibitor (AChEI) is currently used to optimize cognitive benefits, as cholinergic neuronal loss is aggravated by disease progression and AChEls have dose-related cognitive benefits in $A D$ [1-3]. Donepezil $23 \mathrm{mg}$ was approved for the symptomatic pharmacological treatment of moderate to severe AD following a large phase 3 clinical trial showing the efficacy of donepezil $23 \mathrm{mg} /$ day compared with $10 \mathrm{mg} / \mathrm{day}$ [4]. It can be considered in patients with insufficient or waning response to standard doses, however, adverse events (AEs) also increased, which can negatively affect drug compliance and limit the usefulness of donepezil $23 \mathrm{mg}$ [5-9]. In our previous study, we reported that high-dose donepezil ( $23 \mathrm{mg} /$ day) was generally tolerable; this may have been because a slow-release formulation of donepezil $23 \mathrm{mg}$ and dose-titration during the first 4 weeks reduced AEs, particularly nausea, dizziness, and headache [10]. However, donepezil $23 \mathrm{mg}$ still showed more cholinergic AEs than standard-dose donepezil regardless of doseescalation methods, which might be explained by relatively lower body weights (BW) in Asian populations [11-15]. Hence, we hypothesized that lower baseline BW would increase the risks of AEs irrespective of dose-escalation strategies and tried to confirm the relationships between BW and AEs in patients treated with high-dose donepezil.

The objective of this post-hoc analysis was to investigate whether baseline characteristics including BW differ based on reported AEs and which factors might be relevant to the safety of high-dose donepezil.

\section{Methods}

\section{Study design and patient population}

This study was a post-hoc analysis using data from a randomized, multicenter, open-label, parallel group, prospective clinical trial named Optimal Dose Escalation Strategy to Successful Achievement of High Dose Donepezil 23 mg (ODESA). Detailed study design and methods are described in a previous report. ${ }^{1}$ In brief, this study was conducted at 6 centers in South Korea between December 2014 and August 2016 to investigate the safety and tolerability of high-dose donepezil based on doseescalation methods during the first 12 weeks. The study included consecutive patients with moderate to severe AD dementia. Inclusion criteria were as follows: 1) age between 45 and 90; 2) patients clinically diagnosed with probable AD dementia [16]; 3) patients who had been taking donepezil $10 \mathrm{mg}$ daily for at least 3 months prior to screening; 4) a score of 0-20 on the Korean version of the Mini-Mental State Examination (K-MMSE) [17]; 5) a clinical dementia rating (CDR) [18] score $\geq 2$ or a global 
deterioration scale (GDS) [19] score $\geq 4$. Patients with any neurological/ psychiatric disorders that might cause dementias not related to $A D$, severe medical conditions, and previous history of intolerance or hypersensitivity to AChEl were excluded. Participants were randomized into 3 groups: groups 1 and 2 were dose titration groups during the first 4 weeks using 2 different titration methods (group 1: 15mg of donepezil before the escalation; group 2: $10 \mathrm{mg}$ and $23 \mathrm{mg}$ on alternate days before the escalation), and group 3 had no dose titration and directly escalated to $23 \mathrm{mg}$ donepezil. In the current study, the participants were divided into two groups according to the existence of AEs and baseline BW, regardless of the dose escalation methods.

The study protocol and informed consent form were reviewed and approved by the institutional review board of each center. The study was conducted in accordance with the Declaration of Helsinki and principles of Good Clinical Practice.

\section{Safety outcome variables}

Safety and tolerability were assessed at each visit during the study period (baseline and at weeks 4,8 , and 12). The primary safety outcome variable was the incidence of treatment emergent AE of special interests (AESI). We defined 8 most common cholinergic symptoms as AESIs: nausea, vomiting, diarrhea, anorexia, abdominal pain, headache, bradycardia, and weight loss. We assessed physical and neurologic examinations, weight, vital signs, and AE at every study visit. Nausea, vomiting, diarrhea, anorexia, abdominal pain, and headache were assessed by the patients' and caregivers' self-reports. Bradycardia indicates a pulse below 50 at any study visit, and weight loss indicates $a \geq 7 \%$ decrease compared with baseline BW at any time during the study. Other safety outcome variables included drop-out rates, drug compliance (ratio of drug taken to drug prescribed), AE gastrointestinal symptoms (including anorexia, nausea, vomiting, and diarrhea), and any AE occurrence. Subjects who discontinued the study before 12 weeks were asked to complete all end-point assessments at the time of early termination.

\section{Statistical analyses}

Baseline demographic and clinical characteristics were compared using an independent t-test or a Mann-Whitney U test for continuous variables and chi-square tests or Fisher's exact tests for categorical variables. Group comparisons for incidences of AEs were performed using chi-square tests or Fisher's exact tests; to adjust for confounding factors, we also used CochranMantel-Haenzil tests. Relationships between the occurrence of AESIs and baseline characteristics were assessed using binary logistic regression analysis. Using univariable analysis, each baseline factor was assessed separately to measure the relationship. Using multivariable analysis, the five most relevant baseline factors were analyzed together to compare the relationships between baseline characteristics and the occurrence of AESIs. Significance for all tests was set at $a=0.05$, twotailed. All statistical analyses were performed using SPSS 19.0 (SPSS, Chicago, IL, USA).

\section{Results}

\section{Patient enrollment}

Patient enrollment and study design are as described previously [10]. In brief, among 175 patients who were eligible and randomized, we included 160 participants who underwent randomization, took at least one study drug, and underwent at least 1 follow-up evaluation in the safety set population. Fifty participants who discontinued the study due to AEs were also included in the study. We show analyses using the safety set population $(n=160)$ because this study focused on the safety and tolerability of the study drug according to baseline characteristics.

\section{Clinical characteristics according to the occurrence of AESI}

First, we divided all participants into two groups according to the occurrence of AESI, which was the primary safety outcome variable. Participants who experienced AESI $(n=67)$ and those who did not $(n=93)$ were not different with regard to baseline 
characteristics except for BW and body mass index (BMI) (Table 1). Baseline BW (55.90 $\pm .56 \mathrm{~kg}$ in the AESI (+) group vs $59.84 \pm 10.81 \mathrm{~kg}$ in the AESI (-) group, $P=0.011)$ and BMI $(23.27 \pm 3.07 \mathrm{~kg}$ in the AESI (+) group vs $24.30 \pm 3.20 \mathrm{~kg}$ in the AESI (-) group, $P=0.042$ ) were lower in the participants with AESIs (Table 1).

Table 1

Baseline characteristics according to occurrence of adverse events

\begin{tabular}{|c|c|c|c|c|c|c|}
\hline \multirow[t]{3}{*}{ Variables } & \multicolumn{3}{|l|}{ All groups } & \multicolumn{3}{|c|}{ No titration group } \\
\hline & $\mathrm{AESI}^{\mathrm{a}}(+)$ & AESI (-) & $P$ & AESI (+) & AESI (-) & $P$ \\
\hline & $(n=67)$ & $(n=93)$ & & $(n=25)$ & $(n=29)$ & \\
\hline Age, yr & $75.98 \pm 8.77$ & $74.69 \pm 8.63$ & 0.363 & $74.88 \pm 9.94$ & $76.68 \pm 6.27$ & 0.429 \\
\hline Gender, female (\%) & $43 / 67,64.2 \%$ & $55 / 93,59.1 \%$ & 0.519 & $18 / 25,72 \%$ & $16 / 29,55.2 \%$ & 0.202 \\
\hline Group (Titration/no-titration) & $42 / 25$ & $64 / 29$ & 0.718 & $0 / 25$ & $0 / 29$ & $\mathrm{n} / \mathrm{a}$ \\
\hline Education (low/mid/high, n) & $38 / 14 / 15$ & $46 / 27 / 20$ & 0.494 & $15 / 5 / 5$ & $13 / 11 / 5$ & 0.381 \\
\hline Baseline K-MMSE & $13.46 \pm 4.81$ & $13.84 \pm 4.78$ & 0.625 & $15.24 \pm 4.02$ & $13.72 \pm 5.17$ & 0.240 \\
\hline Baseline CDR & $1.67 \pm 0.65$ & $1.65 \pm 0.60$ & 0.917 & $1.56 \pm 0.63$ & $1.76 \pm 0.44$ & 0.296 \\
\hline Baseline GDS & $4.73 \pm 0.62$ & $4.91 \pm 0.69$ & 0.104 & $4.75 \pm 0.61$ & $5.00 \pm 0.77$ & 0.205 \\
\hline Baseline weight $<55 \mathrm{~kg}$ & $30 / 67,44.8 \%$ & $33 / 93,35.5 \%$ & 0.235 & $12 / 25,48 \%$ & $20 / 29,69 \%$ & 0.118 \\
\hline Baseline weight, kg & $55.90 \pm 8.56$ & $59.84 \pm 10.81$ & 0.011 & $53.56 \pm 8.09$ & $60.83 \pm 10.25$ & 0.006 \\
\hline Baseline BMI & $23.27 \pm 3.07$ & $24.30 \pm 3.20$ & 0.042 & $22.64 \pm 2.96$ & $24.48 \pm 3.08$ & 0.030 \\
\hline Donepezil duration (month) & $21.59 \pm 25.55$ & $23.69 \pm 24.46$ & 0.599 & $20.43 \pm 22.44$ & $27.37 \pm 27.83$ & 0.323 \\
\hline \multicolumn{7}{|c|}{$\begin{array}{l}\text { Abbreviations: } K-M M S E \text { Korean version of Mini-Mental State Examination, } C D R \text { Clinical dementia rating, GDS General } \\
\text { deterioration scale, } B M I \text { Body mass index, } A E S I \text { Adverse events of special interest }\end{array}$} \\
\hline
\end{tabular}

Second, we divided participants in the no-titration group $(n=54$, no dose titration during the first 4 weeks of escalation to donepezil 23mg) into two groups according to the occurrence of AESIs. Participants who experienced AESIs $(n=25)$ and those who did not $(\mathrm{n}=29)$ showed similar baseline characteristics except for BW and BMI $(P<0.05$, Table 1$)$. The BW difference was more prominent in the no-titration group $(P=0.006)$ compared to that in all groups $(P=0.011)$. However, baseline BW did not differ between patients with and without AESIs in the dose-titration groups ( $n=106$, dose-titration groups during the first 4 weeks of escalation to donepezil $23 \mathrm{mg}$, data not shown).

\section{Relationship between AE and BW}

Risks of AESIs according to baseline clinical factors including age, gender, group allocation, baseline cognitive status, educational levels, donepezil medication duration, and BW were measured (Table 2). Using the most relevant factors from the univariate analyses, multivariable analyses were performed to identify the most relevant factors related to the occurrence of AESIs. In both the all groups $(n=160)$ and the no-titration group $(n=54)$, BW stood out as the only significant factor related to the occurrence of AESIs (odds ratio $0.953, P=0.020$ in all groups; odds ratio $0.890, P=0.009$ in the no-titration group, Table 2); patients with higher BW had a lower risk of AESIs at 12 weeks from the initiation of high dose donepezil. However, there were no significant baseline factors related to AESIs in the dose-titration groups (Table 2). 
Table 2

Baseline factors related to the occurrence of adverse events

\begin{tabular}{|c|c|c|c|c|c|c|c|c|c|c|c|c|}
\hline \multirow[t]{3}{*}{ Variables } & \multicolumn{4}{|c|}{ All groups $(n=160)$} & \multicolumn{4}{|c|}{ Dose-titration groups $(n=106)$} & \multicolumn{4}{|c|}{ No-titration group only $(n=54)$} \\
\hline & \multicolumn{2}{|c|}{ Univariable } & \multicolumn{2}{|c|}{ Multivariable } & \multicolumn{2}{|c|}{ Univariable } & \multicolumn{2}{|c|}{ Multivariable } & \multicolumn{2}{|c|}{ Univariable } & \multicolumn{2}{|c|}{ Multivariable } \\
\hline & OR & $P$ & OR & $P$ & OR & $P$ & OR & $P$ & OR & $P$ & OR & $P$ \\
\hline Age & 1.018 & 0.361 & 1.010 & 0.634 & 1.039 & 0.116 & 1.036 & 0.175 & 0.973 & 0.423 & & \\
\hline Gender & 1.238 & 0.519 & 1.236 & 0.610 & 1.061 & 0.884 & & & 2.089 & 0.205 & 0.558 & 0.462 \\
\hline $\begin{array}{l}\text { Body } \\
\text { weight }\end{array}$ & 0.960 & $0.016^{*}$ & 0.953 & $0.020 *$ & 0.979 & 0.299 & 0.984 & 0.456 & 0.918 & $0.011 *$ & 0.890 & $0.009 *$ \\
\hline $\begin{array}{l}\text { Baseline } \\
\text { GDS }\end{array}$ & 0.666 & 0.105 & 0.648 & 0.110 & 0.701 & 0.262 & 0.478 & 0.091 & 0.591 & 0.203 & 0.462 & 0.115 \\
\hline $\begin{array}{l}\text { Titration } \\
\text { group } \\
\text { allocation }\end{array}$ & 0.761 & 0.419 & 0.710 & 0.563 & 1.033 & 0.934 & & & & & & \\
\hline $\begin{array}{l}\text { Baseline } \\
\text { K-MMSE }\end{array}$ & 0.984 & 0.623 & & & 0.937 & 0.121 & 0.915 & 0.141 & 1.077 & 0.241 & & \\
\hline Education & 0.906 & 0.622 & & & 0.667 & 0.321 & & & 0.810 & 0.555 & & \\
\hline $\begin{array}{l}\text { Donepezil } \\
\text { duration }\end{array}$ & 1.000 & 0.597 & & & 1.000 & 0.959 & & & 1.000 & 0.320 & & \\
\hline \multicolumn{13}{|c|}{ Abbreviations: GDS General deterioration scale, K-MMSE Korean version of Mini-Mental State Examination } \\
\hline $\begin{array}{l}\text { Logistic re } \\
\text { adverse ev }\end{array}$ & $\begin{array}{l}\text { ssion a } \\
s \text { of } s p\end{array}$ & $\begin{array}{l}\text { alysis us } \\
\text { ial inter }\end{array}$ & $\begin{array}{l}\text { g univ } \\
\text { t (AESI }\end{array}$ & ate and & ultivari & le anal & is was & erforme & 1. Advers & events i & icates & \\
\hline
\end{tabular}

\section{Safety and tolerability according to body weight: all groups}

Additionally, we divided all participants into two groups: the low BW group (baseline BW $<55 \mathrm{~kg} ; \mathrm{n}=63$ ) and the high BW group (baseline BW $\geq 55 \mathrm{~kg}, \mathrm{n}=97$ ). Baseline demographics and clinical characteristics were similar between the two groups except for age and gender distribution when using all groups (Supplementary Table 1). Participants with low baseline BW were older (mean age: $77.1 \pm 8.0$ ) and tended to be female (81\%) than those with high BW group (mean age: $74.1 \pm 8.9$ and female $48.5 \%$ ) (Supplementary Table 1). Therefore, AEs were compared between low and high BW groups adjusted for age and gender differences. Participants in the low BW group showed numerically higher incidences of AEs and drop-out rates due to AEs independent of age and gender differences, although it did not reach statistical significance (Supplementary Table 2). We also compared baseline factors between high $\mathrm{BMI}$ group $(\mathrm{BMI} \geq 23)$ and low $\mathrm{BMI}$ group $(\mathrm{BMI}<23)$, however baseline characteristics and AESI occurrences were not different between the two groups $(P>0.05$, data not shown).

\section{Safety and tolerability according to body weight in the no-titration group}

Participants in the no-titration group ( $n=54$ ) were divided into the low BW group (baseline BW < 55kg; $n=22$ ) and the high BW group (baseline BW $\geq 55 \mathrm{~kg}, \mathrm{n}=32$ ) to assess AEs according to baseline BW. Baseline clinical characteristics are listed in Supplementary Table 1; participants with low BW tended to be female $(81.8 \%, P=0.017)$. Hence, AEs were compared between the low and high BW groups adjusted for gender distribution. Participants in the low BW group showed numerically higher incidences of AEs and drop-out rates due to AEs independent of gender difference, although it did not reach statistical significance (see Supplementary Table 3). We also compared baseline factors between high BMI group (BMI $\geq 23$ ) and low $\mathrm{BMI}$ group $(\mathrm{BMI}<23)$ in the no-titration group, however baseline characteristics and $\mathrm{AESI}$ occurrences were not different between the two groups $(P>0.05$, data not shown). 


\section{Discussion}

We conducted a post-hoc analyses of a 12 week, multicenter, randomized, three-arm prospective clinical trial investigating the safety and tolerability of high-dose donepezil $(23 \mathrm{mg})$. In this study, we assessed whether the participants who experienced AESIs differed from those without AESIs with regard to baseline clinical characteristics; we also investigated baseline factors correlated with the occurrence of AESIs.

Our study showed two main findings. First, baseline BW was lower in participants who experienced AESIs compared to those that did not. Patients with higher BW might have a lower risk of AEs during the first 12 weeks after dose escalation to $23 \mathrm{mg} /$ day. Consistently, BW was the most relevant factor related with the occurrence of AEs. Second, dose titration during the first 4 weeks might weaken the relationship between BW and AESI occurrences, while direct escalation to donepezil $23 \mathrm{mg}$ might strengthen the negative association between BW and AESI. This relationship between BW and AEs is consistent with previous studies which reported that patients with low BW or BMI showed more AEs and poorer tolerability after dose escalation to donepezil $23 \mathrm{mg}[8,11,20]$. However, our study differs from the previous results in some important ways. In our data, binary classification using a cut-off value of BW $55 \mathrm{~kg}$ did not show a statistically significant difference with regard to AEs, though there was some numerical difference. We also focused on AESIs consisting of the eight most common cholinergic AEs related to the use of acetylcholinesterase inhibitors; in addition, we measured relationships between baseline factors and the occurrence of AESIs according to dose escalation methods. As a result, dose-titration is assumed to weaken the inverse relationship between BW and AESI occurrence, possibly by preventing a sharp increase in the peak concentration of high dose donepezil.

Generally, low BW increases the risk of AEs, which seem to be more prominent in cases of direct dose escalation to high-dose donepezil [21, 22]. In the low BW group, the maximal concentration might be increased, time to maximal concentration might be decreased, and drug clearance might be delayed based on the fact that BW can explain hepatic clearance variability and metabolic rate $[23,24]$. In our study, BW turned out to be more sensitive than BMI in predicting the occurrence of AEs. BW seems to be more relevant to the risk of AEs associated with the use of cholinesterase inhibitors at least in Asian demented people. ${ }^{12}$ It is interesting that AESI incidence is similar across the different age and gender groups; in spite of age and gender imbalance, these factors might not influence AESI occurrences. Failure to reach statistical significance using binary classification in the current study might also be explained by other confounding factors such as comorbidities, nutritional status, or other socioeconomic variables that could not be assessed, although we tried to adjust for age and gender differences.

Our study had some limitations that should be considered when interpreting the results. First, we studied a relatively small sample size and included only Korean dementia patients with moderate to severe AD, which could limit the generalizability of our results. Given that the safety in a US-based population was somewhat different from the non-US population [11], safety outcomes related to BW might require cautious interpretation when generalizing to other populations. Second, we did not investigate long-term safety outcomes. However, considering that the incidences of AEs dropped rapidly after the first 4 weeks in previous trials using high dose donepezil [8], and given that patients with moderate to severe dementia might have higher rates of physical disabilities and comorbidities, conducting longer follow-ups would be difficult in this population. Despite these study limitations, our study does reveal an inverse relationship between baseline BW and the occurrence of AE based on the first clinical trial investigating the efficacy of dose-titration prior to escalating to donepezil $23 \mathrm{mg}$. To our knowledge, this is the first evidence showing that dose-titration before escalation to high dose donepezil might weaken these negative associations.

\section{Conclusion}

In this study, baseline BW was the most important factor related to cholinergic AEs during the first 12 weeks of high-dose donepezil regardless of dose-escalation method. Among moderate to severe AD patients considering dose escalation to $23 \mathrm{mg} /$ day, patients with low BW might experience an increased risk of cholinergic AEs. Hence, stepwise dose-titration is 
recommended prior to direct escalation to the high dose in order to enhance drug adherence and to weaken the inverse relationship between $B W$ and the risk of $A E s$, particularly in $A D$ patients with low $B W$.

\section{Abbreviations}

AD: Alzheimer's disease; AChEl: acetylcholinesterase inhibitor; AE: adverse events; BW: body weights; ODESA: Optimal Dose Escalation Strategy to Successful Achievement of High Dose Donepezil 23 mg; CDR: clinical dementia rating; GDS: global deterioration scale; AESI: AE of special interests; BMI: body mass index

\section{Declarations}

\section{Acknowledgments}

Not applicable

\section{Authors' contributions}

JHL and SYK contributed to the study concept and design. YJH and HJK analyzed and interpreted the results. YJH and JHL drafted the manuscript. HJH, YCY, KWP, DWY, SYK, HJK, MSK, and JHL were involved in data collection, recruitment and evaluation of the patients. All authors read and approved the final manuscript.

\section{Funding}

This research was supported by a grant (\# 2014-0576) from the Asan Institute for Life Sciences, Asan Medical Center

\section{Availability of data and materials}

The datasets used and/or analyzed during the current study are available from the corresponding author on reasonable request.

\section{Ethics approved and consent to participate}

All eligible patients who had signed the consent form were included in the study. The study protocol was validated by the Asan Medical center's ethics committee, Seoul, South Korea.

\section{Consent for publication}

Not applicable.

\section{Competing interests}

The authors declare that they have no competing interests.

\section{References}

1. Kuhl DE, Minoshima S, Frey KA, Foster NL, Kilbourn MR, Koeppe RA. Limited donepezil inhibition of acetylcholinesterase measured with positron emission tomography in living Alzheimer cerebral cortex. J Nucl Med. 2000;41:1879-87. 
2. Boehnen NI, Kaufer DI, Hendrickson R, Ivanco LS, Lopresti BJ, Koeppe RA, et al. Degree of inhibition of cortical acetylcholinesterase activity and cognitive effects by donepezil treatment in Alzheimer's disease. J Neurol Neurosurg Psychiatry. 2005;76:315-9.

3. Sabbagh M, Cummings J. Progressive cholinergic decline in Alzheimer's disease: consideration for treatment with donepezil $23 \mathrm{mg}$ in patients with moderate to severe symptomatology. BMC Neurol. 2011;11:21.

4. Farlow MR, Salloway S, Tariot PN, Yardley J, Moline ML, Wang Q, et al. Effectiveness and tolerability of high-dose (23 $\mathrm{mg} / \mathrm{d}$ ) versus standard dose $(10 \mathrm{mg} / \mathrm{d})$ donepezil in moderate to severe Alzheimer's disease: a 24 week, randomized, double-blind study. Clin Ther. 2010;32:1234-51.

5. Ferris S, Cummings J, Christensen D, Doody R, Farlow M, Sabbagh M, et al. Effects of donepezil 23 mg on Severe Impairment Battery domains in patients with moderate to severe Alzheimer's disease: evaluating the impact of baseline severity. Alzheimer's Res Ther. 2013;5:12.

6. Sabbagh M, Cummings J, Christensen D, Doody R, Farlow M, Liu L, et al. Evaluating the cognitive effects of donepezil 23 $\mathrm{mg} / \mathrm{d}$ in moderate and severe Alzheimer's disease: analysis of effects of baseline features on treatment response. BMC Geriatr. 2013;13:56.

7. Farlow M, Veloso F, Moline M, Yardley J, Brand-Schieber E, Bibbiani F, et al. Safety and tolerability of donepezil $23 \mathrm{mg}$ in moderate to severe Alzheimer's disease. BMC Neurol. 2011;11:57.

8. Tariot P, Salloway S, Yardley J, Mackell J, Moline M. Long-term safety and tolerability of donepezil $23 \mathrm{mg}$ in patients with moderate to severe Alzheimer's disease. BMC Res Notes. 2012;5:283.

9. Doody RS, Geldmacher DS, Farlow MR, Sun Y, Moline M, Mackell J. Efficacy and safety of donepezil 23 mg versus donepezil $10 \mathrm{mg}$ for moderate-to severe Alzheimer's disease: a subgroup analysis in patients already taking or not taking concomitant memantine. Dement Geriatr Cogn Disord. 2012;33:164-73.

10. Hong YJ, Han HJ, Youn YC, Park KW, Yang DW, Kim S, et al. Safety and tolerability of donepezil 23 mg with or without intermediate dose titration in patients with Alzheimer's disease taking donepezil $10 \mathrm{mg}$ : a multicenter, randomized, openlabel, parallel-design, three-arm, prospective trial. Alzheimers Res Ther. 2019;11:37. doi:10.1186/s13195-019-0492-1.

11. Salloway S, Mintzer J, Cummings JL, Geldmacher D, Sun Y, Yardley J, et al. Subgroup analysis of US and non-US patients in a global study of high-dose donepezil $(23 \mathrm{mg})$ in moderate and severe Alzheimer's disease. Am J Alzheimers Dis Other Demen. 2012;27:421-32.

12. Lee JH, Sevigny J. Effects of body weight on tolerability of rivastigmine transdermal patch: a post-hoc analysis of a double-blind trial in patients with Alzheimer disease. Alzheimer Dis Assoc Disord. 2010;25:58-62.

13. Cummings JL, Geldmacher D, Farlow M, Sabbagh M, Christensen D, Betz P. High-dose donepezil (23 mg/day) for the treatment of moderate and severe Alzheimer's disease: drug profile and clinical guidelines. CNS Neurosci Ther. 2013;19:294-301.

14. Han SH, Lee JH, Kim SY, Park KW, Chen C, Tripathi M, et al. Donepezil 23 mg in Asian patients with moderate-to-severe Alzheimer's disease. Acta Neurol Scand. 2017;135:252-6.

15. Homma A, Atarashi H, Kubota N, Nakai K, Takase T. Efficacy and Safety of Sustained Release Donepezil High Dose versus Immediate Release Donepezil Standard Dose in Japanese Patients with Severe Alzheimer's Disease: A Randomized, Double-Blind Trial. J Alzheimers Dis. 2016;52:345-57.

16. McKhann GM, Knopman DS, Chertkow H, Hyman BT, Jack CR Jr, Kawas CH, et al. The diagnosis of dementia due to Alzheimer's disease: recommendations from the National Institute on Aging-Alzheimer's Association workgroups on diagnostic guidelines for Alzheimer's disease. Alzheimers Dement. 2011;7:263-9.

17. Han C, Jo SA, Jo I, Kim E, Park MH, Kang Y. An adaptation of the Korean mini-mental state examination (K-MMSE) in elderly Koreans: demographic influence and population-based norms (the AGE study). Arch Gerontol Geriatr. 2008;47:30210.

18. Morris JC. The Clinical Dementia Rating (CDR): current version and scoring rules. Neurology. 1993;43:2412-4. 
19. Reisberg B, Ferris SH, de Leon MJ, Crook T. The global deterioration scale for assessment of primary degenerative dementia. Am J Psychiatry. 1982;139:1136-9.

20. Lee C, Lee K, Yu H, Ryu SH, Moon SW, Han C, et al. Adverse Events with sustained-release donepezil in Alzheimer disease: Relation to body mass index. J Clin Psychopharmacol. 2017;37:401-4.

21. Lee JH, Jeong SK, Kim BC, Park KW, Dash A. Donepezil across the spectrum of Alzheimer's disease: dose optimization and clinical relevance. Acta Neurol Scand. 2015;131:259-67.

22. Sabbagh M, Han S, Kim S, Na HR, Lee JH, Kandiah N, et al. Clinical Recommendations for the Use of Donepezil 23 mg in Moderate-to-Severe Alzheimer's Disease in the Asia-Pacific Region. Dement Geriatr Cogn Dis Extra. 2016;6:382-95.

23. Anderson BJ, Holford NH. Mechanism based concepts of size and maturity in pharmacokinetics. Annu Rev Pharmacol Toxicol. 2008;48:303-32.

24. Fagerholm U. Prediction of human pharmacokinetics-evaluation of methods for prediction of hepatic metabolic clearance. J Pharm Pharmacol. 2007;59:803-28.

\section{Supplementary Files}

This is a list of supplementary files associated with this preprint. Click to download.

- Supplementarymaterial.pdf 